ArtefaCToS. Revista de estudios de la ciencia y la tecnología

eISSN: $1989-3612$

Vol. 10, No. 2 (2021), 2. ${ }^{\text {a }}$ Época, 153-174

DOI: https://doi.org/10.14201/art2021102153174

\title{
Valores y motivaciones que guían la práctica científica en Frankenstein
}

\section{Values and Motivations Guiding Scientific Practice in Frankenstein}

\section{Elenia DENIA}

Department of Philosophy and Cultural Heritage,

Università Ca' Foscari de Venecia, Italia

elenadenia@icloud.com

Recibido: 05/03/2021. Revisado: 27/08/2021. Aceptado: 19/10/2021

\section{Resumen}

La novela Frankenstein o El moderno Prometeo (1818) es una fuente de información abundante sobre la imagen de la ciencia en la sociedad de principios del siglo XIX que permite revelar aspectos nítidos de la dimensión social de la investigación científica de la época. El propósito de este trabajo es, tras contextualizar algunos elementos clave del entorno científico coetáneo a la obra y la educación que recibió Mary Shelley — su autora—, realizar un análisis exhaustivo del texto original para revelar características de la práctica científica del momento, en particular los valores y motivaciones que guían el ejercicio de la ciencia en la narración. El análisis revela que: (1) los valores que caracterizan la práctica científica en el universo Frankenstein son: rigor; altruismo; empeño y perseverancia; determinación; valentía; imaginación; serenidad; reconocimiento; y responsabilidad; mientras que (2) las motivaciones para emprender la actividad científica son: curiosidad e interés; pasión o entusiasmo; ambición; y voluntad. La caracterización de la labor científica a partir de la obra exhibe una conceptualización de lo que se considera buena ciencia que parece vigente en el imaginario de la ciencia actual.

Palabras clave: Frankenstein; educación científica; imagen de la ciencia; percepción social de la ciencia; divulgación científica; educación inclusiva; estudios de género; literatura de ciencia ficción. 


\begin{abstract}
The novel Frankenstein or The Modern Prometheus (1818) is a rich source of information on the image of science in early nineteenth-century society, revealing clear aspects of the social dimension of scientific research at the time. The purpose of this paper is, after contextualising the scientific environment at the time of the work and the education that Mary Shelley, its author, received, to carry out an exhaustive analysis of the original text in order to reveal characteristics of the scientific practice of the time, in particular the values and motivations that guide the practice of science in the narrative. The analysis reveals that: (1) the values that characterise scientific practice in the Frankenstein universe are: rigour; altruism; endeavour and perseverance; determination; courage; imagination; serenity; recognition; and responsibility; while (2) the motivations for undertaking scientific activity are: curiosity and interest; passion or enthusiasm; ambition; and will. The characterisation of scientific work derived from the book exhibits a conceptualisation of what is considered good science that seems to be current in the imaginary of science today.
\end{abstract}

Keywords: Frankenstein; science education; image of science; social perception of science; science popularization; inclusive education; gender studies.

\title{
1. Introducción
}

La ficción es uno de los modos de difusión del hecho científico (Moreno Castro, 2011, 110) y, en particular, la literatura destaca "por su potencialidad para sensibilizar y provocar reflexiones acerca de las transformaciones generadas por el avance científico-tecnológico" (Sannazzaro, 2012, 186). En ese sentido hay consenso en que la novela Frankenstein o El moderno Prometeo (1818), escrita por Mary Shelley, invita a reflexionar sobre los peligros del poder emergente que representa, si no la ciencia en sí, al menos su mala utilización por parte de científicos irresponsables, tema que será recurrente a partir de entonces en la literatura. La obra ha sido descrita como "la historia del hombre de ciencia que, con consecuencias catastróficas, busca conquistar la naturaleza, rivalizar con la divinidad y crear una nueva vida, y quien luego niega el amor a la vida que ha creado" (Abrams, 2006, 956). Al respecto, puede señalarse que Frankenstein ha sido ampliamente considerada como la obra fundacional del género de la ciencia ficción: Shelley introdujo en su obra "un elemento de un futuro posible, y con ello transformó para siempre las posibilidades de la literatura" (Scholes y Rabkin, 1982, 17).

La idea de Frankenstein se gestó en la Villa Diodati, a orillas del lago Leman en Suiza, cuando un grupo de jóvenes intelectuales se reunió en 1816 para pasar el verano más frío del que se tiene registro. Al otro lado del globo, en Indonesia, el volcán Tambora había entrado en erupción el pasado año, dejando la atmósfera del hemisferio norte de la Tierra cubierta de cenizas y gas durante un largo 
período tiempo. La finca había sido alquilada por el emblemático poeta Lord Byron y allí hospedó a la joven Mary Wollstonecraft — de 18 años de edad-y a su pareja, el poeta romántico Percy Shelley, además de a su médico y asistente John William Polidori, y a la hermanastra de Mary, Claire Clairmont, quien mantuvo una aventura con el anfitrión. Aquel fue conocido como el año sin verano. La lluvia incesante los obligaba a confinarse en la vivienda durante días y a veces se entretenían junto al fuego leyendo historias de fantasmas. Durante el encuentro los inquilinos se animaron a escribir una historia de terror, siendo los resultados más significativos de aquel reto narrativo el relato El vampiro (1819) de Polidori y la obra Frankenstein o El moderno Prometeo (1818) de Mary Shelley. Esta última constituye el objeto de estudio del presente trabajo.

Si bien la novela se publicó de forma anónima en 1818 con un prólogo escrito por Percy Shelley — por aquel entonces ya se habían casado y ella había adoptado su apellido-, pronto se reconoció la autoría de Mary y el libro gozó de gran popularidad, llegando a estudiarse en escuelas secundarias y universidades y siendo aclamado entre lectores de todo tipo (Myers, 2000).

A pesar de tratarse de una pieza de ficción, parece razonable pensar que la novela describe de forma fidedigna la concepción de la ciencia coetánea a la obra, de acuerdo con — como se expone más abajo- los datos biográficos de la autora y el propio contenido científico que puede extraerse del texto, incluyendo la transición intelectual de su protagonista, Víctor Frankenstein, quien gracias a la formación universitaria deja atrás a autores ocultistas y los sustituye por lecturas de ciencia moderna, a su entender mucho más rigurosa.

De forma general, el interés del público por la ciencia se ve reflejado ya desde el siglo XVIII en el elevado número de obras de divulgación científica y de conferencias públicas — que tenían lugar no solo en grandes ciudades como París o Londres sino también en balnearios y ciudades de descanso como Bath-e incluso en cafeterías (coffee houses) que hacían de aulas de divulgación científica, así como en la creciente creación de sociedades científicas (Malet, 2002).

Diversos aspectos científicos tratados en el texto por la autora confirman sus conocimientos sobre los debates científicos del momento - por ejemplo, relativos a expediciones científicas, experimentos de anatomía o dudas morales respecto a los usos de la ciencia y la tecnología- (Ruiz-Castell, 2021, 2-3). Nótese además que la obra fue revisada en 1831 en una edición que incluía una introducción de la autora que resulta esclarecedora, brindando algunas declaraciones sobre cómo concibió la historia.

Ciertamente, la obra de Shelley es un ejemplo de cómo las representaciones de la actividad científica que encontramos en la literatura pueden servir para instruir y cautivar al público en relación con determinados planteamientos científico-tecnológicos, así como para reflexionar sobre sus aspectos éticos y estimular determinadas investigaciones. (Ruiz-Castell, 2021, 6) 
El presente artículo examina la imagen de la ciencia que transmite la obra Frankesntein y trata de averiguar, a través del análisis de la narración, cuáles son las motivaciones del protagonista para dedicarse a la ciencia y los valores que guían la práctica científica en el libro, entendida hoy en día como un ejercicio que se desarrolla en comunidad (Vinck, 2015) bajo ciertos criterios éticos. Nótese que se repara en la dimensión social de la investigación científica que puede extraerse de la lectura del relato, asumiendo que el mismo puede revelar tendencias relacionadas con la ciencia en la época, como por ejemplo cuestionamientos morales respecto al uso de la ciencia y la tecnología.

De forma adicional, puede ser de utilidad recordar la propuesta de Robert Merton del ethos cientifico para valorar si las normas del buen hacer científico postuladas explícitamente por el sociólogo en el siglo XX ya estaban presentes por aquel entonces, ya que, para numerosos académicos, se trata de una conceptualización que sigue vigente en el imaginario de la ciencia actual y que por tanto puede servir de referencia a efectos comparativos entre la obra de Shelley y la práctica científica de nuestros días. En cualquier caso, más allá de identificar unos rasgos que faciliten una lectura actual del proceder científico de entonces, en este artículo no se pretende ignorar que la ciencia de desarrolla en contextos sociohistóricos específicos - en efecto, desde la desde la filosofía de la ciencia es comúnmente aceptada la idea formulada por Thomas Kuhn de que la práctica científica no es ahistórica (Kuhn, 1962)—.

En concreto, la célebre propuesta desarrollada por Merton (1942) se centra en el sistema de organización social de la ciencia y propone una serie de valores y normas éticas que rigen la actividad científica, "ese complejo, con resonancias afectivas, de valores y normas que se consideran obligatorios para el hombre de ciencia” (Merton, 1968, 357). Según Merton, las normas que rigen la conducta del científico son: (1) el universalismo, para el que la ciencia se rige por criterios universales o impersonales; (2) el comunalismo, de modo que las ideas de la ciencia son bienes que pertenecen a la comunidad científica, producidos en, por y para la sociedad; (3) el desinterés, dado que se investiga para el beneficio común de acuerdo con valores altruistas e integridad, no por intereses particulares; y (4) el escepticismo organizado, que se presenta, por ejemplo, en la evaluación sistemática de las producciones científicas por la comunidad. Más tarde también añade (5) el reconocimiento como valor, centrado en la originalidad de los descubrimientos inéditos y en los principios de humildad al reconocer el trabajo de predecesores y colegas, así como señalar las limitaciones del propio (Merton, 1968). Este último valor es el único que admite asociar un nombre al descubrimiento científico.

Para llevar a cabo el análisis, primero conviene detenerse en el marco que contextualiza la obra, que incluye aspectos de la formación de Mary Shelley, debates científicos del momento entre las élites ilustradas del siglo XIX y elementos científicos presentes en la narración de forma explícita. Estos aspectos se tratan en los siguientes epígrafes. 
Como pincelada previa, puede precisarse que la etimología del título completo de la novela ofrece algunas pistas sobre su contenido al hacer referencia a Prometeo, un personaje de la mitología griega que osa robar el fuego de los dioses para entregar poderes divinos a los hombres y es castigado por Zeus. El moderno Prometeo es encarnado por el aprendiz de filósofo natural protagonista de la historia, Víctor Frankenstein.

Debe puntualizarse que, si bien en aquel entonces no se utilizaba el término cientifico sino filósofo natural u hombre de ciencia, sí se hará uso del mismo a lo largo de este texto por simplicidad y para facilitar comparaciones con los roles actuales de los científicos. Conviene matizar que no fue hasta 1833 cuando el pensador William Whewell acunó el término cientifico — scientist- para designar a los filósofos naturales (Encyclopædia Britannica, 1911).

\section{Educación de Mary Shelley}

Siendo hija de intelectuales comprometidos con los derechos de las mujeres, Mary Shelley recibió una formación poco convencional. Fue criada por su padre, William Godwin, un reconocido filósofo político de la época que la instruyó bajo los criterios de la educación femenina racional desarrollados por su esposa y que incluían nociones de ciencia y experimentos sencillos. En sus escritos, la obra de la feminista Mary Wollstonecraft — fallecida poco después de dar a luz a Maryaboga por la educación de las hijas (Wollstonecraft, 1787) y pone de manifiesto que las estudiantes de primaria deberían hacer los mismos experimentos científicos que los escolares varones de la misma edad (Wollstonecraft, 1792).

Durante las primeras décadas del siglo XIX, además de desarrollarse debates sobre el papel social de la ciencia y la adecuación de la inclusión de la experimentación científica en la formación propia de un caballero, también se discutió la conveniencia de educar - $\mathrm{O}$ no- a las niñas en disciplinas científicas. En particular, se popularizaron libros de enseñanza de las ciencias para niñas escritos por mujeres divulgadoras y se ha especulado sobre la posible influencia en Mary de los mismos. Aunque no existe evidencia directa, para algunos parece probable que Mary hubiera aprendido con estos textos didácticos de ciencia para niñas en los que aparecían maestras que dialogaban con sus alumnas (Fara, 2008). Las autoras de estos manuales participaron en el esfuerzo colectivo de dar a conocer una ciencia con presencia social creciente, y aunque su público objetivo eran las niñas, también llegaron a niños y jóvenes. Además, con su recurso al diálogo ficticio, colocaron a las mujeres mentoras en el lugar de los hombres, una posición de autoridad que serviría de modelo para las niñas. Paralelamente, la inclusión de sermones morales en los textos tranquilizó a los padres, inquietos sobre la conveniencia de que las niñas emprendieran actividades científicas.

La mayoría de los activistas a favor de la educación femenina de gran influencia en la intelectualidad de la época, entre ellos Erasmus Darwin —abuelo de 
Charles Darwin-, estuvieron de acuerdo en que las mentes de las mujeres eran diferentes de las de los hombres y menos aptas para el trabajo metódico requerido en la ciencia por su naturaleza emocional, y en que determinadas materias como la botánica o la historia natural eran más adecuadas para las nińas que otras como la física o la química. No obstante hubo autoras que desafiaron estas convenciones; por ejemplo, Margaret Bryan escribió un Compendio de los Sistemas de Astronomía ([1797] 2012) y Jane Marcet redactó conversaciones sobre química que influenciaron a Michael Faraday, padre del electromagnetismo, quien aprendió esta disciplina leyendo sus textos después del trabajo - aprendiz de encuadernador- (Bahar, 2001).

En el caso de Mary, debe mencionarse que recibió una formación que además de las asignaturas tradicionales para nińas incluía materias de ciencias como geografía, matemáticas y química, pero de acuerdo con los parámetros de la época, según los cuales educar a las mujeres en ciencia las mejoraría como esposas y madres, sin ser el objetivo convertirlas en damas cientificas porque esta condición las volvería problemáticas y desagradables, según el dialogo imaginario de la escritora Mary Edgeworth (Edgeworth, [1825] 2010; Fara, 2008).

También es conocido que Godwin recibía en su casa con frecuencia la visita de otros intelectuales, entre ellos eminentes hombres de ciencia que influirían en la obra de la autora. Entre estas visitas, pueden destacarse las de algunos filósofos naturales distinguidos de Londres (Robinson, 2017); como el investigador y divulgador científico William Nicholson, a quien recurría para pedir información sobre las últimas teorías en química, física, óptica, biología y otras ciencias naturales (St. Clair, 1991), o el químico y también divulgador Humphrey Davy, quien fue a casa de Godwin por primera vez, de joven, acompañado de Samuel Coleridge, otro influyente intelectual inglés que comparó la labor de este químico con la del poeta, al buscar la unidad de la sustancia a través de una multiplicidad de formas (Hindle, 1990, 30, como se citó en Knight, 1972). Davy fue el autor de Elements of chemical philosophy (1812), un libro que Mary leyó en el otońo de 1816 según anotó en su diario, mientras ideaba la historia de Frankenstein —de hecho, consta que Percy Shelley lo había encargado en Londres a su librero el año en que se publicó (Burdiel, 2007)—.

\section{Contexto científico de la obra}

No es de extrañar la abundancia de referencias a la actualidad científica de la época en la obra de Mary Shelley, ya que en el ambiente de cientifismo que reinaba en el siglo XIX la divulgación de las ideas científicas gozó de una gran popularidad entre la élite ilustrada, con Gran Bretańa y Francia como primeras potencias. Los avances económicos y sociales propiciaron el crecimiento de una clase media culta, cada vez más alfabetizada y mejor retribuida (Nieto-Galán, 
2006). A ello contribuyeron las exhibiciones de ciencia y las colecciones particulares, así como las nuevas tecnologías de impresión, que abarataron los libros y las publicaciones periódicas, e impulsaron su circulación masiva.

En particular, las charlas de temática científica, concebidas para suscitar el interés y el entusiasmo del público lego, tuvieron una amplia difusión ya desde el siglo XVIII y a lo largo del siguiente, siendo impartidas tanto por científicos de primer nivel como por otros conferenciantes más modestos; por ejemplo, profesores no universitarios artesanos que explicaban cuestiones prácticas como el funcionamiento de instrumentos científicos (Malet, 2002). A ellas asistían tanto miembros de las clases altas y medias como trabajadores con el deseo de adquirir conocimientos que mejorasen su situación social. Para las clases altas inglesas, la alfabetización de los trabajadores constituía además un instrumento para «llevar a las masas el gusto y el beneficio moral del conocimiento; querían desvelar la mano de Dios en la Naturaleza; querían, al exponer el mundo como un sistema organizado y ordenado, mantener a las clases trabajadoras en su lugar» (Gregory y Miller, 2000, 23). Un ejemplo destacado lo constituyen las sesiones públicas de la Royal Institution de Londres — fundada en 1799—, que desde los primeros ańos puso en marcha las conferencias y cursos para difundir el conocimiento científico mediante coloquios filosóficos y exhibiciones de distinta índole -y que, de hecho, suponían su mayor fuente de ingresos-.

En el caso de Mary Shelley, hay constancia de que asistió a algunos de estos espectáculos científicos. Por un lado, según recoge Godwin en su diario, llevó a su hija a algunas de las conferencias sobre química y anatomía impartidas a principios de 1812 (Godwin, 2010). Por otro lado, existen registros en el propio diario de Mary de que fue a una de estas conferencias en Londres el 28 de diciembre de 1814, en el Theatre of Grand Philosophical Recreations, titulada Electricidad, gas, aeroestación, fantasmagoría y deportes hidráulicos (Robinson, 2017), en la que se incluían exhibiciones de química y electricidad.

También debe mencionarse el éxito que gozaban algunas obras científicas en aquella época, al cautivar la imaginación de gran parte de la sociedad británica y que, por tanto, también parece razonable vincular a las mismas el interés por la ciencia de Mary. Por ejemplo, los libros escritos por el citado químico Humphrey Davy, en los cuales ya se había contemplado la idea de convertir la materia muerta en viva (Rodríguez y Baños, 2014, 40). Otro antecedente que podría citarse es la obra de divulgación de Joseph Priestley Historia de la Electricidad (1767) - y su versión más pedagógica, Una Introducción Familiar al Estudio de la Electricidad (1768)—, que impulsó algunas exhibiciones ya a finales del siglo XVIII con experimentos científicos en vivo dirigidos al público general.

Sobre la posible influencia del fenómeno de la electricidad en el proceso de dotar de vida a la criatura — tratado en el análisis posterior del presente artículo-, pueden señalarse los populares experimentos de Luigi Galvani, quien "había acuñado el término electricidad animal para describir cualquier cosa que 
activara los músculos de las ranas cuando pasaba una corriente a través de sus patas" (Kitson, 2017, 350). Estos experimentos desembocaron en otros efectuados por su sobrino y discípulo Giovanni Aldini, quien organizó sesiones multitudinarias en las que infundió estímulos eléctricos a cerebros de animales muertos - e incluso al cerebro de un criminal decapitado - para producir movimientos — por ejemplo en labios u ojos_-, lo que sin duda resultó muy llamativo para las personas ilustradas de la época (Finger, 2000, 111). No es de extrańar que en la literatura romántica se cuestione el carácter polémico de la ciencia a la vez que se ensalce su poder transformador. La novela refleja esta tensión, por ejemplo, al relatar el robo de cadáveres, una práctica común en la época debido a la escasez de cuerpos para su estudio en escuelas de anatomía (Ruiz-Castell, 2021, 3).

Conviene aclarar que en la época no había una postura antagonista entre hombres de letras y hombres de ciencias: como en siglos anteriores, la ciencia era una parte de la cultura intelectual de la humanidad (Lucas, 1979). No obstante, debe admitirse que resulta problemático definir lo que se entendía por ciencia en aquel período, dado que Frankenstein fue publicada con anterioridad a la consolidación de la práctica científica como actividad profesionalizada y dividida en disciplinas, que se produciría en la segunda mitad del siglo (Vinck, 2015). En cualquier caso, existían sociedades científicas que se autodenominaban «Literary and Philosophical» (Malet, 2002) —en referencia a la filosofía natural—y había publicaciones periódicas que daban cuenta de la conexión entre ciencia y artes, como por ejemplo el Journal of Natural Philosophy, Chemistry and the Arts (Hindle, 1990). En efecto, los conocimientos científicos formaban parte del acervo cultural de la sociedad ilustrada, e incluso los aficionados podían practicar la ciencia: "La ciencia en la primera mitad del siglo XIX como en siglos anteriores, era una parte de la cultura intelectual de la humanidad, en la que todos podían entrar y de la que todos podían beneficiarse" (Lucas, 1979, 330).

Por último, otro hecho relevante que corrobora el interés de Mary por la ciencia, es que aquel verano de 1816 le fabricó un globo aerostático a su amado Percy, además de regalarle un telescopio por su cumpleaños (Marshall, 1889).

\section{La ciencia en Frankenstein}

En sí misma, la novela Frankenstein es un testimonio indiscutible de que a Mary Shelley le interesaban los temas científicos y, de hecho, prestando atención al contenido de la obra, destaca que se trata de una novela científicamente actualizada - al menos atendiendo al hecho de que su autora era conocedora de los debates científicos más interesantes de la época (Ruiz-Castell, 2021).

A continuación se exhiben algunos ejemplos que ratifican el interés de Mary por la ciencia. 
En primer lugar, la narración comienza con el personaje de Robert Walton, quien desea explorar las variaciones magnéticas de la brújula y emprende un viaje en busca de un pasaje por el ártico, en gran parte motivado por descubrir el Polo Norte Magnético. Llama la atención la similitud de la travesía con la del capitán Constantine Phipps, de la Royal Navy, quien encabezó una expedición hacia el Polo Norte en 1773 y que probablemente fue el predecesor que inspira el viaje de este personaje (Kitson, 2017).

Otra pista que encontramos en el texto es la declaración de Víctor cuando llega a Matlock con su mejor amigo, Henry Clerval, afirmando que visitan "los pequeńos gabinetes de historia natural, donde las muestras están dispuestas del mismo modo que aparecen en las colecciones de Servox y Chamonix" (Shelley, [1818] 2017, 203). Este fragmento resalta el conocimiento de Mary sobre los gabinetes de curiosidades, concebidos con carácter expositivo como habitaciones llenas de objetos que mostraban las maravillas de la ciencia y la tecnología - y que ya eran populares desde el siglo XVI- Estas exposiciones estaban dirigidas a un público de clase alta y a estudiantes, además de servir como centros de investigación para eruditos y científicos (Montañés, 2011).

Se ha sugerido también que los experimentos de Alphonse Frankenstein, padre de Víctor, están basados en un acontecimiento que le relataría Percy sobre la construcción de una cometa eléctrica para almacenar electricidad en botellas de Leyden (Robinson, 2017).

Respecto a la idea de crear vida a partir de materia muerta, según se ha señalado reiteradamente en diversos análisis de la obra, la autora pudo verse influenciada por las ideas sobre la reanimación de cadáveres extendidas en la época, constituyendo lo que para algunos indicaría una visión mecanicista del mundo. No obstante, si bien es cierto que en numerosas ocasiones se ha argumentado que Mary fue influenciada por el galvanismo, éste no aparece mencionado en la historia de forma explícita, ni tampoco el uso de la electricidad en el experimento de Víctor. Sin embargo, hay una frase en el texto que, aunque sutil, puede interpretarse en esa dirección, cuando el protagonista despierta a su criatura y resuelve: "coloqué a mi alrededor la maquinaria para la vida con la que iba a poder insuflar una chispa de existencia en aquella cosa exánime que estaba tendida a mis pies" (Shelley, 2017, 88). Cabría preguntarse si esa chispa de existencia alude a una chispa de electricidad para animar el sustrato biológico de su experimento.

Es en el prólogo incluido en la versión revisada de 1831, cuando Mary menciona el galvanismo - "Quizá un cadáver podría reanimarse; el galvanismo había dado pruebas de cosas semejantes: quizá se podrían fabricar las partes componentes de una criatura, reunidas y dotadas de calor vital" (Shelley, 1982)—. Además en él la autora se describe a sí misma como una oyente silenciosa de las conversaciones entre Percy Shelley y Lord Byron, quienes discutían sobre la naturaleza del principio de la vida y sobre los experimentos de Erasmus Darwin, por ejemplo contando que conservó fideos - vermicelli - en un caja de vidrio hasta que por 
algún medio extraordinario aquello comenzó a moverse con movimiento voluntario. Respecto a este fenómeno, Mary manifiesta su desconfianza e incide en la diferencia entre lo que Darwin consiguió y lo que se dice que consiguió — que, en cualquier caso, no desembocó en una entidad viva—, trazando así una línea clara entre realidad e imaginación.

Sumado a ello, ya en el prefacio del libro de 1818 escrito por Percy Shelley, la historia se sitúa en el contexto de la filosofía natural contemporánea, desechando el componente sobrenatural de forma explícita. También el propio Víctor expresa en el relato que "Durante mi educación, mi padre había tomado todo tipo de precauciones para evitar que mi mente se viera impresionada por terrores sobrenaturales. [...] un cementerio no era para mí más que un conjunto de cuerpos privados de vida" (Shelley, [1818] 2017, 78). Pese a ello, algunos autores han defendido que la creación de vida artificial en Frankenstein responde a viejas leyendas (Rieger, [1974] 1982), aunque la postura comúnmente defendida en los análisis más recientes de la obra es que responde a preocupaciones filosóficas y científicas muy vivas en el momento (Pollin, 1965; Vasbinder, 1984), que además fueron reseñadas en las publicaciones periódicas de la época, como el incipiente debate científico sobre si en la electricidad se encontraba el origen de la vida.

Las inquietudes de la autora coinciden, en efecto, con las de los intelectuales de su tiempo, y en este sentido aunque la novela Frankenstein no presta atención a lo sobrenatural, sí especula con los posibles desarrollos futuros de las ideas científicas de la época; de ahí que sea considerada por muchos como la obra fundacional del género de la ciencia ficción. Como nota de interés, también puede señalarse que el texto obvia cualquier referencia a la religión, habiendo una única mención a Dios que hace la criatura, de forma retórica, al final de la novela.

Por otra parte, es interesante la sugerencia de que una conocida disputa que tuvo lugar en 1814 entre el científico John Abernethy y su discípulo William Lawrence pudo inspirar las discrepancias relatadas por Víctor con sus profesores de universidad (Robinson, 2017). Se sabe que Percy Shelley conocía personalmente a ambos (Bieri, 2008), puesto que asistió a clases del primero - que concebía la vida como "una sustancia invisible, móvil, sutil, análoga, por un lado, al alma, y por el otro, a la electricidad” (Butler, 1996, 304)— mientras que el segundo fue su médico — quien proporcionaba una explicación materialista de la vida-.

Otros autores abogan por que el mentor más influyente en la carrera de Víctor, el profesor Waldman, es un personaje inspirado en un profesor de Percy Shelley en Eton, el doctor James Lind (Goulding, 2002, 257), un médico que fue miembro de un distinguido club de caballeros interesados en ciencia llamado Lunar Society, creado a finales del siglo XVIII en Inglaterra y al que pertenecían algunos intelectuales muy populares como James Watts, Erasmus Darwin o 
Joseph Priestley (Robinson, 2017). Un dato curioso es que Lind había realizado experimentos de estimulación neuromuscular en ranas empleando electricidad, algo que pudo haber compartido con Percy (Rodríguez y Baños, 2014, 40).

Nótese, por último, que el apellido Frankenstein pudo estar inspirado por el del médico y botánico alemán de mediados del siglo XVII Frank von Frankeneau, quien investigó la potencial reanimación de tejidos biológicos (Burdiel, 2007). Otros, en cambio, lo atribuyen al médico y físico alemán del siglo XVIII Christian Gottlieb Kratzenstein, cuyo apellido también presenta similitudes con el del personaje (Kaplan, 2002, 577-578).

\section{Análisis de las motivaciones y valores que guían la práctica científica en la novela}

Al comienzo de la novela, el explorador Robert Walton escribe una serie de cartas a su hermana, relatando su travesía hacia el Polo Norte, que recuerdan a las narraciones de viajes de la época. Una de las ideas que se extrae de este pasaje es la de ser el primero en enfrentar lo desconocido, propio del carácter intrépido del explorador así como del científico, cuando se llevan a cabo investigaciones de frontera, siendo Walton un personaje que desea tener la primicia a la hora de desentrañar los misterios del magnetismo terrestre y de las observaciones astronómicas.

Allí podré descubrir la maravillosa fuerza que atrae la aguja de la brújula, y podré comprobar miles de observaciones celestes que precisan solo que se lleve a cabo este viaje para conseguir que todas sus aparentes contradicciones adquieran coherencia para siempre. Saciaré mi ardiente curiosidad cuando vea esa parte del mundo que nadie visitó jamás antes y cuando pise una tierra que no fue hollada jamás por el pie del hombre. (Shelley, [1818] 2017, 41-42)

Nótese además que una de las motivaciones que impulsan el viaje es la curiosidad. Durante su aventura, Walton se encuentra con un hombre moribundo de acento extraño llamado Víctor Frankenstein, quien le relata su triste historia; la de un hombre de ciencia caído en desgracia. De este relato se estudian, a continuación, los elementos que definen el aprendizaje y la práctica de la ciencia en la obra, esencialmente reflejados en el primer tercio del libro.

Atraído por la búsqueda de la piedra filosofal y el elixir de la vida, si bien un Víctor Frankenstein adolescente se muestra inspirado por antiguos ocultistas y alquimistas - Cornelio Agripa, Paracelso y Alberto Magno-, durante el relato se disculpa aduciendo que él no pertenecía a una familia científica ni había asistido a clase en Ginebra, por lo que puede desprenderse que el protagonista aprende a demarcar la ciencia con las enseñanzas científicas modernas, otorgándole un valor de conocimiento superior e incluso exhibiendo cierta vergüenza respecto a 
sus ídolos de la adolescencia. En el texto, Víctor expresa de forma manifiesta el sentido de la maravilla por la ciencia, y en sus palabras se percibe una transformación en el pensamiento del personaje cuando comprende que la ciencia comporta progreso y admite que los autores esotéricos que admira quedan desfasados a la luz de las técnicas científicas modernas para explicar fenómenos y para experimentar:

Los fenómenos naturales que tienen lugar todos los días delante de nuestros ojos no me pasaban desapercibidos. La destilación, de la cual mis autores favoritos eran absolutamente ignorantes, me causaba asombro, pero con lo que me quedé maravillado fue con algunos experimentos con una bomba de aire que llevaba a cabo un caballero al que solíamos visitar. La ignorancia de mis filósofos en estas y muchas otras disciplinas sirvieron para desacreditarlos a mis ojos... pero no podía apartarlos a un lado definitivamente antes de que algún otro sistema ocupara su lugar en mi mente. (Shelley, [1818] 2017, 66)

Como nota adicional, resulta aquí llamativa la concepción implícita de Víctor del progreso científico, ya que el fragmento invita a pensar que necesita disponer de un sistema mejor que explique el mundo antes de desechar el anterior completamente, aunque el mismo le resulte dudoso - un planteamiento que recuerda a las ideas desarrolladas por Thomas Kuhn en el siglo XX (Kuhn, 1962)—.

En particular, el protagonista asegura que aprender sobre el fenómeno de la electricidad fue decisivo para derribar a los autores esotéricos, por lo que parece que el concepto tiene un peso especial en la obra, al demarcar de un chispazo la ciencia moderna de creencias esotéricas. Víctor cuenta que a los 15 años, durante una fuerte tormenta mientras pasa las vacaciones en su casa familiar de Belrive, ve como un rayo cae a 20 metros sobre un roble y lo carboniza. Respondiendo a sus preguntas, su padre le habla de la electricidad e incluso realiza algunos experimentos para mostrarle sus efectos; entre ellos, prepara "una cometa con una cuerda y un cable que podía extraer aquel fluido desde las nubes" (Shelley, [1818] 2017, 67).

Dado su creciente interés por los fenómenos de la ciencia, Víctor decide acudir en una ocasión a una clase, en Ginebra, de una asignatura en curso. En ella se siente abrumado por los tecnicismos del lenguaje científico y reacciona con rechazo hacia los estudios, aduciendo que le desagrada una ciencia que solo consiste en palabras, pues el joven no es capaz de asociar ideas a los conceptos propios de la química — potasio, boro, sulfatos, óxidos-. Este episodio lo hace abandonar su intención de instruirse en la filosofía natural, que sin embargo retoma más adelante, cuando ingresa en la universidad, aunque para entonces exhibe cierta evolución en sus lecturas al decantarse hacia autores de mayor rigor científico como el Conde de Buffon. En este proceso, la respuesta emocional del protagonista oscila entre la frustración hacia la complejidad de la ciencia y la admiración hacia la misma a la hora de explicar fenómenos. 
Ya a los 17 ańos, Víctor viaja a la Universidad de Ingolstadt y asegura que "deseaba ardientemente adquirir más conocimientos" (Shelley, [1818] 2017, 72). Una vez allí, cuando el aprendiz de filósofo natural se presenta ante el profesor Krempe, de nuevo admite que no se ve motivado por el estudio, en este caso porque le resulta desagradable su aspecto físico, calificándolo de profesorcillo vanidoso. Más adelante admitirá, sin embargo, encontrar cierta valía y sabiduría en las clases de Krempe, lo que sugiere que el personaje madura y desarrolla respeto hacia el contenido del programa de enseñanza más allá de sus reservas hacia el profesor, por lo que parece que le atribuye cierta objetividad al contenido de las lecciones. Sobre las lecturas preuniversitarias de Víctor, en su primer encuentro, el profesor lo reprende:

Tiene usted el cerebro atestado de sistemas caducos y nombres inútiles. ¡Dios mío...! ¿En qué desierto ha estado viviendo usted? ¿Es que no había un alma caritativa que le dijera a usted que esas tonterías que ha devorado con avidez tienen más de mil años y son tan rancias como anticuadas? No esperaba encontrarme a un discípulo de Alberto Magno y de Paracelso en el siglo de la Ilustración y la ciencia. Mi querido señor, deberá usted comenzar sus estudios absolutamente desde el principio. (Shelley [1818] $2017,72)$

Hasta ahora, Víctor había saciado sus intereses de forma autodidacta, con algunos progresos en sus lecturas y en el aprendizaje sobre fenómenos naturales pero con cierto desorden teórico, sin embargo el profesor Krempe le advierte de que la carrera de un hombre de ciencia necesita guía y disciplina, y que a medida que el conocimiento avanza, se desechan algunas teorías por falta de pruebas que son sustituidas por otras más perfeccionadas.

A pesar de la actitud despreciativa por parte del profesor, Víctor no desecha del todo a sus antiguos ídolos porque considera que sus fines son legítimos, aunque sí se acrecienta su desconfianza hacia estos autores caducos al tiempo que los ensalza, al considerar dignos sus objetivos. Esto le lleva a reflexionar sobre los propósitos que mueven la práctica científica y se siente contrariado, porque al iniciarse en sus estudios de filosofía natural moderna encuentra que la materia ha sufrido un gran cambio: "la ambición del investigador parecía limitarse a rebatir aquellos puntos de vista en los cuales se fundaba principalmente mi interés en la ciencia" (Shelley, [1818] 2017, 73), proporcionando una concepción de la ciencia dedicada en gran medida a la refutación — una idea que recuerda a las contribuciones sobre el funcionamiento de la ciencia propuestas posteriormente por el filósofo Karl Popper (Popper, 1962)—.

Con cierta indignación, Víctor describe la actividad científica como una labor minuciosa cuyo proceder es sistemático y que deja de lado las grandes cuestiones que antańo guiaron a otros filósofos. Sin embargo, aún conservando esa concep- 
ción sobre la forma de proceder de la ciencia moderna, cuando asiste a la lección del profesor Waldman, éste le proporciona un nuevo prisma, cautivándolo con su intelecto y elegancia.

Los antiguos maestros de la ciencia — dijo— prometían imposibles y no consiguieron nada. Los maestros modernos prometen muy poco. Saben que los metales no pueden transmutarse y que el elixir de la vida es solo una quimera. Pero estos filósofos, cuyas manos parecen hechas solo para escarbar en la suciedad y cuyos ojos parecen solo destinados a escudrińar en el microscopio o en el crisol, en realidad han conseguido milagros. Penetran en los recónditos escondrijos de la Naturaleza y muestran cómo opera en esos lugares secretos. Han ascendido a los cielos y han descubierto cómo circula la sangre y la naturaleza del aire que respiramos. Han adquirido nuevos y casi ilimitados poderes: pueden dominar los truenos del cielo, simular un terremoto, e incluso imitar el mundo invisible con sus propias sombras. (Shelley, [1818] 2017, 74-75)

Ese proceder sistemático de la práctica científica es descrito por este profesor, entre elogios, como un camino heroico hacia las verdades ocultas de la naturaleza. Además, en la última frase, Waldman enfatiza su discurso dando cuenta de los poderes que proporciona el conocimiento, y presentando a la ciencia como un medio para dominar la naturaleza - una idea heredada de las obras de Francis Bacon y René Descartes (Bacon, 1620; Descartes, 1637) — y para reproducir e imitar sus fenómenos en entornos controlados.

Cuando Víctor visita al profesor Waldman en su casa, pronto siente gran afinidad hacia su interlocutor, quien lo toma como discípulo y le aconseja que no descuide otras ciencias de la filosofía natural para no convertirse en un mero experimentador — como por ejemplo las matemáticas — atribuyendo así valor al trabajo teórico y proporcionando una idea de la ciencia más completa, constituida por un conjunto de áreas que se complementan. En el profesor encuentra no solo a un mentor sino también a un amigo, una declaración que invita a pensar que no existe una clara línea divisoria entre la vida privada y la profesión de docente académico, yendo la relación profesor-alumno más allá de lo estrictamente académico. Además, dado que la visita se produce en la vivienda particular de Waldman, el relato parece sugerir que la vida personal y el ejercicio de la enseñanza no están completamente diferenciados.

A partir de entonces, Víctor modifica su visión sobre la ciencia y considera que los libros de investigadores modernos que le ha recomendado Waldman están repletos de genialidades. Curiosamente, llega a afirmar que siente predilección por la química solo porque le agrada el atractivo del profesor, aunque a medida que profundiza en el estudio de la ciencia asegura que le interesa más como un fin. Se siente atraído así por los objetivos de la ciencia y no tanto por la praxis, pero se dispone a trabajar intensamente hasta altas horas en el laboratorio y progresa a pasos agigantados. Habla entonces de la pasión y la fascinación que 
ejerce la ciencia sobre aquellos que la han experimentado, asegurando además que "Una inteligencia de capacidad mediana que se empeña con pasión en un estudio necesariamente alcanza un gran dominio en dicha disciplina" (Shelley [1818] 2017, 78). Esto sugiere que tanto el empeño como el trabajo metódico deben ser garantes del oficio. Además, también atribuye al científico la cualidad de la valentía cuando pregunta retóricamente: “¿cuántas cosas podríamos descubrir si la cobardía o el desinterés no entorpecieran nuestras investigaciones?" (Shelley [1818] 2017, 78).

Sumado a ello, otra de las reflexiones que ofrece Víctor acerca de la carrera científica es que para él la riqueza es un asunto menor, sin embargo sí manifiesta reiteradamente en el texto un interés por la fama, y de esto se desprende que la labor científica no está concebida en Frankenstein para generar beneficios económicos cuantiosos pero sí prestigio o notoriedad, por lo que podríamos extraer los valores del altruismo - al menos en cierto grado- - y del reconocimiento en el trabajo científico.

Una vez establecidas las motivaciones del protagonista respecto a la práctica de la ciencia y adquiridos ciertos conocimientos, el moderno Prometeo se pregunta dónde reside el principio de la vida y formula su proyecto científico, una búsqueda inspirada por los autores que leía con anterioridad a su formación universitaria. Para comprender ese principio trabaja con fruición, profana tumbas para hacerse con los cadáveres y estudiar la muerte — concebida como la corrupción del cuerpo humano- y también tortura animales; calificando su tarea como una penosa labor que acarrea horrores pero cuyo balance es positivo en el cómputo global de sus resultados. Si bien en ocasiones fracasa, eso no le impide proseguir; por lo que otro elemento que caracteriza la práctica científica sería la perseverancia. A pesar de ser un trabajo incómodo, Víctor cree que está siguiendo el camino correcto mediante el cual logra desentrańar los misterios de la naturaleza - aquellos recónditos escondrijos-, determinación que le proporciona un entusiasmo infatigable.

En cierto momento del proceso, el protagonista muestra sus reservas ante el trabajo que lo ocupa y asoma el primer amago de duda, no obstante continúa con su quehacer declarando que es un trabajo odioso pero irresistible. A pesar de que la búsqueda del conocimiento debería, a su parecer, requerir serenidad y no dejarse llevar por impulsos, su justificación para continuar es que la culpa es de la disciplina en sí - llegando a calificarla de perjudicial e impropia de la mente humana-. De forma determinante para la historia, durante el desarrollo de la actividad científica, Víctor Frankenstein no asume la responsabilidad sobre su trabajo, ni siquiera se la plantea, porque parece que éste responde a un orden superior. Sin embargo, ese dilema sobre asumir o no su responsabilidad como creador se presenta después y perdura hasta el final de la obra, sentando la base sobre la que se sostiene el motor de la historia. 
Más que como un artista, se percibe a sí mismo como un esclavo que, de alguna forma, pierde el alma o la sensibilidad cegado por su meta. Para lidiar con la repugnante tarea aparta sus sentimientos y afectos durante el periodo de investigación, que durará dos angustiosos años y que requerirá su confinamiento, pensando que al terminar volverá a la normalidad. Dos aspectos interesantes del proceso son que, en su etapa de aislamiento, no comparte ideas con sus colegas científicos y además descuida su aspecto físico, por lo que parece encarnar esa imagen del científico aislado y desaliñado dedicado en exclusiva a sus investigaciones que tantas veces se ha evocado desde el siglo XX. Mientras investiga admite que, al no tratarse de un fin conseguido y completo, se siente motivado para continuar experimentando, aunque vacila sobre cuál puede ser el modo de utilizar ese poder.

Víctor desea avanzar en su labor con rapidez, y para ello, más allá de realizar una tarea debidamente perfeccionada, agiliza el proceso al componer una versión humana de grandes dimensiones que resulta menos costosa, mientras fantasea con las alabanzas de los seres creados que cree merecer. Cuando su labor culmina e insufla vida a la criatura, la contempla y en ella ve un engendro abominable, una especie de momia, declarándose incapaz de soportar su aspecto físico e incluso apelando a un poema que habla de un demonio, asignando así maldad a la fealdad. Tras abandonar inmediatamente a la criatura a su suerte, el científico se convierte en un ser enajenado, aún más antisocial y que emite risotadas nerviosas. Manifiesta entonces una antipatía hacia la filosofía natural, admite que lo ha aniquilado un objetivo egoísta y reniega de la ciencia.

Su amigo Clerval, quien va a pasar una temporada a Ingolstadt, lo encuentra gravemente aquejado y desmejorado. Como nota de interés, Víctor señala, al reparar en su amigo, que la imaginación de Clerval es demasiado vívida como para dedicarse a las ciencias, reflexión que se omite en la edición del libro de 1831, por lo que podría desprenderse que, tras pensarlo, la autora no excluye a la imaginación como rasgo inherente al quehacer científico.

De nuevo, cuando el moderno Prometeo se reencuentra con la criatura en la montaña helada en Suiza, dispuesto a batirse, ésta le ruega clemencia y que escuche su triste historia, y es ahí cuando Víctor admite por primera vez que siente que tiene deberes hacia su creación. La criatura presenta emociones delicadas pero alerta de que quiere infundir el mal ante la falta de afecto que tanto aborrece, pero a condición de no hacerlo, le pide a su creador que fabrique una compañera para él. Tras aceptar el pacto, Víctor se siente incapaz de vencer su repugnancia hacia la idea de emprender dicha tarea, pero se determina a llevarla a cabo. Resulta especialmente llamativo, no obstante, que para entonces el protagonista admite haber olvidado los procedimientos exactos empleados para crear al monstruo, es decir, el método científico, alegando que se ha desvanecido de su 
cabeza al haberse concentrado en el resultado - "descubrí que era incapaz de componer una mujer sin volver a dedicarle muchos meses de estudio y laboriosas pruebas" (Shelley, [1818] 2017, 193)—. Además:

Recordé también la necesidad perentoria en que me hallaba, bien de viajar a Inglaterra, bien de entablar una larga correspondencia con los filósofos de ese país, cuyos conocimientos y descubrimientos me resultaban indispensables en semejante empresa. Esta última forma de conseguir la información precisa era lenta y enojosa. (Shelley, [1818] 2017, 195)

Con este pasaje se resalta la importancia de la cooperación entre científicos y la idea de que la ciencia se sostiene sobre el trabajo de muchos, progresando con lentitud - aunque en ocasiones, como ya se ha puesto de relieve, un científico aislado también pueda hacerla avanzar-. Emprende así un viaje con Clerval, en quien ve reflejado lo que él mismo había sido antaño; "era curioso y estaba deseando adquirir nuevas experiencias y conocimientos" (Shelley, [1818] 2017, 201). Sobre su amigo, en particular comenta que "su inteligencia se crecía cuando se encontraba en compañía de hombres de talento, y descubrió en sí mismo una capacidad y unas emociones superiores a las que habría sospechado cuando se encontraba con personas menos inteligentes" (Shelley, [1818] 2017, 204). En este sentido Víctor da a entender de nuevo que la compañía de personas ilustradas favorece el desarrollo de la ciencia y potencia el carácter científico de sus practicantes.

Una vez se encuentra en condiciones de emprender de nuevo la actividad científica, el protagonista vuelve a recluirse, esta vez durante unos meses, en una cabaña aislada en el norte de Escocia, en las islas Orcadas, para fabricar una compañera para el monstruo; una tarea que no logrará concluir vencido por el horror. Cuando le relata su historia a Robert Walton, la postura de Víctor frente al descubrimiento científico ha cambiado radicalmente:

[...] vea cuán peligrosa es la adquisición de conocimientos y cuánto más feliz es el hombre que acepta su lugar en el mundo en vez de aspirar a ser más de lo que la naturaleza le permitirá jamás. (Shelley, [1818] 2017, 80)

Efectivamente, al final de la novela, Víctor Frankenstein insta a Robert Walton a evitar la osadía y la ambición por el éxito en los descubrimientos científicos.

\section{Conclusión}

En el presente artículo se recogen una serie de observaciones sobre las características de la práctica de la ciencia extraídas del texto original de la obra Frankenstein, esencialmente reflejadas en el primer tercio del libro, el cual se centra tanto en describir las motivaciones del protagonista cuando va a estudiar ciencias como en el proceso de aprendizaje que culmina con un proyecto científico propio y las consecuencias que genera su desarrollo. 
La ciencia en Frankenstein se presenta como una actividad para comprender los fenómenos naturales, fruto del trabajo teórico y de la experimentación y que progresa por acumulación de conocimientos, operando en las fronteras del saber para desvelar los misterios del mundo y revelar sus verdades ocultas. Es objetiva, rigurosa y tiene un alto grado de complejidad —emplea, por ejemplo, numerosos tecnicismos-. A través de su práctica, los fenómenos de la naturaleza se pueden simular, imitar e incluso dominar.

Si bien Mary pudo tener acceso a libros ocultistas en la biblioteca de su padre (Pulido, 2012, 15) - y, de hecho, son los que inicialmente captan la atención del personaje-, la novela no constituye un collage de ideas ocultistas e ideas científicas como algunos han sugerido (Burdiel, 2007, 60-61), dado que la educación universitaria de Víctor deriva en que deje atrás nociones pseudocientificas marcando un antes y un después en su concepción de los mecanismos de la vida.

De acuerdo con la obra, para que se produzca progreso científico se necesitan, por un lado, conocimientos previos, y por el otro, o bien la cooperación entre agentes para agilizar el ejercicio de la ciencia a través del intercambio de correspondencia entre investigadores o propiciando encuentros entre los mismos, o bien el confinamiento del científico, pues el desarrollo aislado también puede acelerar el proceso. La actividad científica sería así una labor que puede ejercerse mediante la inquebrantable voluntad de un científico que se aísle del mundo para trabajar sin descanso - en el caso de Víctor, prepara un taller para tal efecto en dos ocasiones-, pero cuyo desarrollo también puede impulsarse de forma favorable cuando se sostiene sobre el trabajo de una red de científicos - hombres ilustrados que se aconsejan y se asesoran en comunidad-, denotando una estructura social en la que sus integrantes cooperan e incluso compiten entre sí.

Además la práctica requiere una responsabilidad individual. Idealmente el investigador debe establecer unos objetivos legítimos para su trabajo, aunque la meta pueda cegarlo y dejarse llevar por fines egoístas, como le sucede a Víctor con su afán por la fama. Nótese que, en efecto, el éxito de la actividad científica confiere prestigio y reconocimiento al investigador.

Respecto a la investigación científica, se presenta como una práctica minuciosa y sistemática, y en parte su forma de proceder consiste en rebatir supuestos. Puede llevarse a cabo con una inteligencia moderada, empeño y trabajo metódico, siendo una actividad más sistemática que creativa y que requiere perseverancia - aunque también cierta imaginación-. Se trata, no obstante, de un trabajo laborioso y lento, por lo que en ocasiones el tedio puede enojar a su practicante.

En términos generales, los rasgos asociados a la práctica científica que se han identificado a lo largo del análisis son: (1) las motivaciones para emprender el ejercicio de la ciencia: curiosidad e interés; pasión o entusiasmo; ambición; voluntad; y (2) los valores que guían su práctica: rigor; altruismo; empeño y perseverancia; 
determinación; valentía; imaginación; serenidad; reconocimiento; y responsabilidad. En particular, la reflexión sobre la responsabilidad es el resultado de todo el desarrollo de la obra.

Respecto a las motivaciones que caracterizan al científico extraídas del texto, puede incidirse en que son comunes a otras actividades creativas humanas y no específicas de la dedicación a la ciencia, quizá siendo la curiosidad la más distintiva de la práctica científica. Esto corrobora que las motivaciones que impulsan la práctica de la ciencia no son diferentes a las de otras actividades culturales, una idea que se ha señalado reiteradamente en diferentes estudios sociales de la ciencia.

Cabe destacar, por otro lado, que el cumplimiento de los valores no es imperativo, puesto que la historia refleja que se puede hacer ciencia sin que el investigador cumpla todo el conjunto, quebrantando por ejemplo, el de la serenidad, como declara Víctor, y por supuesto, el de la responsabilidad — fuente de su posterior agonía - Por tanto, puede haber cierta flexibilidad en la labor del científico sin que el avance de la ciencia se vea afectado, y en este sentido los valores identificados constituirían una guía para la buena ciencia.

Cabría preguntarse, a continuación, si los valores extraídos del universo Frankenstein, que se asumen representativos del imaginario colectivo del momento, difieren de aquellos que guían la práctica científica en la actualidad bajo los criterios de lo que se considera buena ciencia. Si uno piensa en los valores de la buena ciencia en el sentido mertoniano enunciados un siglo después universalismo, comunalismo, desinterés, escepticismo organizado y reconocimiento (Merton, 1968)—, de acuerdo con lo expuesto a lo largo del artículo, tienen una alta correspondencia con los identificados en la obra. Puede extraerse así que el incipiente desarrollo científico de principios del XIX ya tenía presente estas normas de forma implícita que luego serían reflejadas explícitamente en la propuesta del sociólogo y que se siguen considerando acertadas a día de hoy.

Finalmente, si se asume la premisa de partida del artículo, a saber, que el texto de ficción de 1918 revela aspectos sólidos de la percepción de la ciencia —y del científico - de la época, puede verse mediante el análisis de la novela aquí expuesto que es posible proporcionar una imagen nítida sobre cómo se concibe el proceso de producción de conocimiento científico en términos de valores y motivaciones que guían la práctica científica de principios del siglo XIX, al menos desde el punto de vista del sector ilustrado, al que pertenecía Mary Shelley.

\section{Referencias bibliográficas}

Bacon, Francis (\{1620\} 1878). Novum organum scientiarum. In Novum Organum, sive indicia vera de Interpretatione Naturae. En Thomas Fowler (Ed.), Bacon's Novum organum. London: Clarendon Press. 
Bahar, Saba (2001). Jane Marcet and the limits to public science. The British Journal for the History of Science, 34(1), 29-49. https://doi.org/10.1017/S0007087401004289

Bieri, James (2008). Percy Bysshe Shelley. Baltimore: Johns Hopkins University Press.

Bryan, Margaret (\{1797\} 2012). A Compendious System of Astronomy: In a Course of Familiar Lectures. Cambridge: Cambridge University Press.

Burdiel, Isabel (2007). Introducción. En Isabel Burdiel (Ed.), Frankenstein o El moderno Prometeo (1818) (pp. 9-65). Madrid: Cátedra.

Butler, Marilyn (1996). Frankenstein and Radical Science. En J. Paul Hunter (Ed.), Mary Shelley, Frankenstein. New York: Norton.

Descartes, René (1637). Discours de la méthode pour bien conduire sa raison et chercher la vérité dans les sciences. Leiden: Leyde.

Edgeworth, Maria (1861). Harry and Lucy, Concluded. Boston: Crosby, Nichols, Lee and company.

Encyclopædia Britannica (1911). Whewell, William. En Encyclopedia Britannica (11 ed., Vol. 28). Cambridge: Cambridge University Press.

Fara, Patricia (2008). Educating Mary: Women and scientific literature in the early nineteenth century. En Christa Knellwolf y Jane Goodall (Eds.), Frankenstein's Science: Experimentation and Discovery in Romantic Culture, 1780-1830 (pp. 1732). Aldershot y Burlington: Ashgate.

Finger, Stanley (2000). Minds behind the brain: A history of the pioneers and their discoveries. Oxford University Press.

Godwin, William (2010). The diary of William Godwin. En Victoria Myers, David O’Shaughnessy y Mark Philp (Eds.). Oxford: Oxford Digital Library.

Goulding, Christopher (2002). The real Doctor Frankenstein?. Journal of the Royal Society of Medicine, 95(5), 257-259.

Hindle, Maurice (1990). Vital matters: Mary Shelley's Frankenstein and romantic science. Critical Survey, 2(1), 29-35.

Kaplan, Peter (2002). The real Dr Frankenstein: Christian Gottlieb Kratzenstein?. Journal of the Royal Society of Medicine, 95(11), 577-578.

Kitson, Peter J. (2017). The Romantic Period, 1780-1832. En Paul Poplawski (Ed.), English Literature in Context (pp. 306-402). Cambridge: Cambridge University Press. https://doi.org/10.1017/9781316493779

Kuhn, Thomas (1962). The structure of scientific revolutions. Chicago: University of Chicago Press.

Lucas, John Randolph (1979). Wilberforce and Huxley: a Legendary Encounter. The Historical Journal, 22(2), 313-330. http://www.jstor.org/stable/2638867

Malet, Antoni (2002). Divulgación y popularización científica en el siglo XVIII: entre la apología cristiana y la propaganda ilustrada. Quark, 13-23. 
Marshall, Florence Ashton (1889). The Life \& Letters of Mary Wollstonecraft Shelley. London: Richard Bentley \& Son.

Merton, Robert King (1942). Science and technology in a democratic order. Journal of legal and political sociology, 1(1), 115-126.

Merton, Robert King (1968). Social theory and social structure. New York: The Free Press.

Montañés Perales, Óscar (2011). Problemas epistemológicos de la comunicación pública de la ciencia [Tesis doctoral, Universidad de Salamanca]. Biblioteca Virtual Miguel de Cervantes.

Moreno Castro, Carolina (2011). La construcción periodística de la ciencia a través de los medios de comunicación social: hacia una taxonomía de la difusión del conocimiento científico. ArtefaCToS. Revista De Estudios Sobre La Ciencia Y La tecnología, 3(1), 109-130.

Myers, Mitzi (2000). Mary Wollstonecraft Godwin Shelley: The Female Author between Public and Private Spheres. En Betty T. Bennett y Stuart Curran (Eds.), Mary Shelley in Her Times (pp. 160-172). Baltimore, London: Johns Hopkins University Press.

Nieto-Galán, Agustí (2006). Libros para todos: la ciencia popular en el siglo XIX. Quark: Ciencia, medicina, comunicación y cultura, 37, 46-52.

Norton Antology (2012). Mary Wollstonecraft Shelley. En Stephen Greenblatt (Ed.), The Norton Anthology English Literature Volume 2 (Eighth Edition) (pp. 958-971). New York: W. W. Norton \& Company.

Pollin, Burton R. (1965). Philosophical and Literary Sources of Frankenstein. Comparative Literature, 17(2), 97-108. https://doi.org/10.2307/1769997

Popper, Karl (1962). Conjectures and refutations: The growth of scientific knowledge. New York, London: Basic Books.

Pulido Tirado, Genara (2012). Vida artificial y literatura: mito, leyendas y ciencia en el Frankestein de Mary Shelley. Tonos digital: Revista electrónica de estudios filológicos, 23, 1-17.

Rieger, James (1982). Mary Shelley's life and composition of Frankenstein. En James Rieger (Ed.), Frankenstein or, The Modern Prometheus. The 1818 text (pp. 11-24). Chicago, London: University of Chicago Press.

Robinson, Charles (2017). Introducción. En David H. Guston, Ed Finn y Jason Scott Robert (Eds.), Frankenstein. Edición anotada para científicos, creadores y curiosos en general: Bicentenario 1818-2018 (pp. 23-36). Barcelona: Ariel.

Rodríguez, Gemma y Baños, Josep (2014). Frankenstein: un mito más allá del cine de ciencia ficción. Revista de Medicina y Cine, 10(1), 37-44.

Ruiz-Castell, Pedro. (2021). Diálogos entre ciencia y literatura: notas sobre Frankenstein o el moderno Prometeo, de Mary W. Shelley. Acta Scientiarum. Language and Culture, 43, e55061. 
Sannazzaro, Jorgelina (2012). «Black Mirror»: la ciencia ficción como punta de lanza para una reflexión ética de los usos sociales de la tecnología. ArtefaCToS, 5(1), 185-193.

Scholes, Robert y Rabkin, Erik (1982). La ciencia ficción: historia, ciencia, perspectiva. Madrid: Taurus.

Shelley, Mary (\{1818\}2017). Frankenstein. Edición anotada para cientificos, creadores y curiosos en general: Bicentenario 1818-2018. David H. Guston, Ed Finn y Jason Scott Robert (Eds.). Barcelona: Ariel.

Shelley, Mary (\{1831\} 1982). Mary Shelley's Introduction to the Third Edition (1831). En James Rieger (Ed.), Frankenstein or, The Modern Prometheus. The 1818 text. Chicago, London: University of Chicago Press.

St Clair, William (1991). The Godwins and the Shelleys: a Biography of a Family. Baltimore: Johns Hopkins University Press.

Vasbinder, Samuel Holmes (1984). Scientific attitudes in Mary Shelley's Frankenstein. Ann Arbor: UMI Research Press.

Vinck, Dominique (2015). Ciencias y sociedad: sociología del trabajo científico. Barcelona: Gedisa.

Wollstonecraft, Mary (1787). Thoughts on the education of daughters: with reflections on female conduct, in the more important duties of life. London: Joseph Johnson.

Wollstonecraft, Mary (1792). A Vindication of the Rights of Woman: with Strictures on Political and Moral Subjects. London: Joseph Johnson. 